\title{
COMPARATIVE GENETICS OF DROSOPHILA WILLISTONI
}

\author{
B. SPASSKY and TH. DOBZHANSKY \\ Columbia University, New York
}

\section{INTRODUCTION}

Received 26.xii.49

Drosophila willistoni is one of the species which were used in the pioneer studies on comparative genetics of Drosophila. Lancefield and Metz (1922) and Ferry, Lancefield and Metz (1923) reported a number of mutants, published a linkage map of the X-chromosome, and suggested that only about half of the $\mathrm{X}$ of this species corresponds to the X-chromosome of $D$. melanogaster, while the other half of the $\mathrm{X}$ of $D$. willistoni carries genes which are autosomal in D. melanogaster. The work on $D$. willistoni was then abandoned. Sturtevant and Novitski (I94I) re-analysed the published information and concluded that one of the two limbs of the X-chromosome of $D$. willistoni corresponds to the $\mathrm{X}$, and the other limb to the left part of the third chromosome of $D$. melanogaster. The homologies of the other chromosomes remained obscure.

The mutant strains of Metz and his collaborators are no longer in existence. Because $D$. willistoni is very favourable for investigations on the population genetics of tropical species, we initiated in 1944 a new collection of mutants and the construction of linkage maps. The results are reported in the present article.

\section{MATERIAL AND METHOD}

D. willistoni is one of the commonest species of Drosophila in the American tropics, and it is found from the southern tip of Florida to Southern Brazil (the state of Rio Grande do Sul). Strains from the same, and especially from different, geographic regions very often differ by inversions of blocks of genes in their chromosomes. The geographic origin of the material with which the genetic work is done is therefore important. Most of our mutants were obtained in a strain from Belem, Pará, Brazil, which was known to be free of inversions : that is to say it is homogeneous in respect of inversions. Metz and his collaborators worked with a strain from Cuba, which almost certainly differed from ours in gene arrangement. Some of our mutants induced by X-ray treatment proved to be associated with chromosomal aberrations; the same is true for a few mutants which were found in strains of other than Belem origin. These mutants are unfit for construction of linkage maps, except when they are 
freed from association with the chromosomal aberrations by crossing over with the standard Belem chromosomes. Except where otherwise specified in the descriptions of the mutants, the latter are of Belem origin and free of major chromosomal aberrations. The technique of the work with $D$. willistoni has been the same as described in the article by Spassky, Zimmering and Dobzhansky, dealing with $D$. prosaltans and published in the same issue of this journal.

\section{SEX-LINKED MUTANTS}

copper $(c p)$.-March 1945, in an X-rayed culture. Eye colour Brazil red (Ridgway, plate I), age change slight. Testis sheath pale yellow. Posterior scutellar bristles form an angle of about $45^{\circ}$ with the plane of the scutellum. Homozygous females sterile. This combination of traits is not, as far as we know, found in any mutant in other species of Drosophila.

cut $(c t)$. - September 1946, spontaneous. Wing margins notched (fig. I). Resembles cut ${ }^{13}$ in D. melanogaster (fig. 14, Morgan et al., 1925), and probably indicates homology of the loci. May or may not be allelic to beaded of Lancefield and Metz (1922).

echinus (ec).-May 1945, spontaneous. Eyes very rough, facets rounded and very convex, but the rows of the facets fairly regular. Eyes large, slightly bulging. Body build and bristles normal. Possibly this is an allele of rough of Lancefield and Metz (1922). Among the mutants of D. melanogaster, resembles most the second-chromosome recessive, echinoid, rather than the sex-linked echinus.

eosin $\left(w^{e}\right)$.- - June 1944 , in an X-rayed culture. Eye colour between grenadine red (Ridgway, plate II) in young, and pompeian red (plate XIII) in old flies. Teste, colourless. An allele of white. No sexual dimorphism. The eosin-prune compound has eyes about capucine orange (plate III). The orange mutant of Lancefield and Metz (1922) was in all probability also an allele of white.

forked $(f)$.- June 1945, in an X-rayed culture. Bristles shortened and irregularly bent, some of them, particularly the posterior dorsocentrals and the scutellars, forked or singed at the tip in most flies. Resembles some weak alleles of forked in D. melanogaster, and may be homologous. The difficulty of establishing the homology is increased by the existence in our material of a phenotypically very similar, but not allelic, mutant called singed (see below). Sturtevant and Novitski (194I) believed that the stubby mutant of Lancefield and Metz (1922) is a homologue of forked of $D$. melanogaster, but, judging from the description, stubby is rather different from our forked.

Incomplete (Inc).--September 1944, in an X-rayed culture. The fourth and fifth wing veins partly or wholly missing between the crossvein and the margin (fig. I). Viable and more extreme in homozygous than in heterozygous condition, and highly variable in both. The weakest expression differs from wild type only in having the posterior crossvein oblique instead of at right angles to the veins. The strongest expression destroys the crossvein as well as the longitudinal veins distal to it, and a part of the fourth vein proximal to the crossvein. No analogues in other species.

lozenge (lz).-February 1945 , in an X-rayed culture. Eyes smaller than normal, facets rough and fused, the eye surface as though varnished, the 
eye margin lighter than the centre, in some individuals dark specks scattered over the eye. Although less extreme than most alleles of lozenge in $D$. melanogaster, this mutant is probably homologous. Judging from the description, resembles also morula of Lancefield and Metz (1922), but the locus of morula in the chromosome indicated by these authors is quite different from ours (see below).

miniature $(m)$.- March 1945 , in an X-rayed culture. Wings slightly more than half as long as normal (fig. I), of normal proportions, opaque because of the high density of the wing hairs. Females sterile. An excellent parallel of miniature in $D$. melanogaster.

Notch $(\mathcal{N})$.- April 1945, in an X-rayed culture. The third to the fifth longitudinal veins considerably thickened. Thoracic hair disarranged. No notching of the wing margin. Lethal in the male and associated with an inversion which suppresses crossing over from Incomplete to roughoid (fig. 2). Despite the unusual phenotype (absence of notching of the wing margin), probably homologous to Notch in $D$. melanogaster and several other species.

prune $(p n)$.--May I944, in an X-rayed culture. Eye colour coral red (Ridgway, plate XIII) in young flies, rapidly darkening with age to about Morocco red (plate I). Testes yellow but lighter than in normal males. Since this mutant phenotype resembles several sex-linked and autosomal mutants in $D$. melanogaster, the homology is an open question.

reduced veins $(r d v)$.- April I944, in an X-rayed culture of Rio de Janeiro origin. All wing veins strongly reduced (fig. I), except that the costa and a part of the third longitudinal vein are always preserved. Viability fair in the male, but lethal in homozygous females. This is perhaps the most extreme known case of a sexual difference in the viability effect of a mutant in Drosophila. Probably this is an extreme allele of the mutant called short by Lancefield and Metz (1922), and possibly a homologue of veinlet in D. melanogaster.

rose $(r s)$.-November 1946 , spontaneous. Eyes cornelian red to rufous (Ridgway, plate XIV). In the eosin-rose compound, the eye colour light ivory. The mutant was lost before the colour of the testis sheath had been studied. Homology ambiguous; among the mutants in the left limb of the third chromosome of $D$. melanogaster, remotely resembles rose, among the sex-linked mutants, not unlike prune, carnation, or ruby.

roughoid $(r u)$.- March 1945 , in an X-rayed culture. Eyes rough, normal in size and shape, no black erupted facets. A possible parallel to roughoid in $D$. melanogaster.

scute (sc).--August 1945 , in an X-rayed culture. The anterior scutellars, and one or both posterior scutellar bristles missing. A good parallel to scute of $D$. melanogaster. Like scute of Lancefield and Metz (I922).

sepia (se).-April I945, in an X-rayed culture. Eye colour somewhat more brownish and more translucent than normal in young flies, darkening with age to Hay's maroon (Ridgway, plate XIII). Testis sheath bright yellow. Some of the scutellar bristles are missing in homozygous females. Although the eye colour in old flies is lighter than in sepia of D. melanogaster, these mutants are probably homologous.

short $(s h)$.-April 1944, in an X-rayed culture. The fifth vein does not reach the margin. Scutellum with a small but constant nick on the posterior margin between the scutellar bristles. The venation is variable; in some flies the fifth vein is complete but the end section thinner than 
the rest of the vein. Posterior crossvein often arched. Normally recessive but behaves as a dominant in females heterozygous for short and for reduced. No clear parallel in other species; the identically named mutant in D. pseudoobscura probably not homologous.

singed (sn).-April 1945, in an X-rayed culture. Phenotypically indistinguishable from forked (see above), but not allelic. Homozygous female fertile. Probably homologous to either singed or to forked of D. melanogaster, and to forked found by Lancefield and Metz (1922) in D. willistoni.

stubbloid $(s b)$.-April I944, in an X-rayed culture of Rio de Janeiro origin. Bristles about half as long, but as thick or even thicker than normal, tapering at the ends. Wings normal. Homozygous females sterile. Phenotypically resembles very closely stubbloid in the right limb of the third chromosome of $D$. melanogaster; this resemblance is closer than that to javelin in the left limb of the third, and much closer than to kurz or small bristle in the X-chromosome of $D$. melanogaster, although all these mutants reduce the length of the bristles. Clearly differs from stubby of Lancefield and Metz (1 922).

veinlet (ve).-July I 947 , spontaneous. The second, third, and fourth veins, especially the latter two, abbreviated. The fifth vein usually not affected. Almost certainly identical with abbreviated of Ferry, Lancefield and Metz (1923), and doubtfully homologous to veinlet in D. melanogaster. white $(w)$.- January 1945, in an X-rayed culture. Eyes white, testes transparent. Viability poor. An allele of eosin. An excellent homologue of white in $D$. melanogaster.

yellow $(y)$.-March 1947 , in an X-ray culture. Body, bristles, and wings yellow. An excellent parallel to yellow of D. melanogaster.

\section{MUTANTS OF THE SECOND LINKAGE GROUP}

abbreviated $(a b b)$.--March 1944, spontaneous. Some of the macrochaetæ absent, but the basal rings are preserved. Highly variable but does not overlap wild type ; orbitals, postverticals, ocellars, dorsocentral or scutellar bristles may be absent ; head bristles absent more often than those on the thorax. Asymmetry very frequent. Among the mutants of $D$. melanogaster resembles most rasé in the right limb of the third chromosome, much less similar to abbreviated in the second chromosome.

brown (bw).-July 1944, in an X-rayed culture of Rio de Janeiro origin. Eye colour vinaceous-rufous (Ridgway, plate XIV), slightly darker in old flies. Wings usually wavy, but normal in some flies. Testis sheath transparent. The eye colour resembles that in brown of $D$. melanogaster.

Emarginate $(E m)$.-February 1945, in an X-rayed culture of Belem origin. Eyes decreased to about half of normal size, often with a notch on the anterior margin, the facets roughened and disarranged in the vicinity of the notch. Lethal when homozygous. Resembles Emarginate in D. pseudoobscura (third chromosome); homology with Lobe of $D$. melanogaster ( $c f$. Sturtevant and Tan, I937) doubtful.

Hook $(H k)$.- August 1944, in an X-rayed culture of Bertioga origin. Some of the bristles on the thorax or on the scutellum hooked or bent at right angles, most often near the tip. Some bristles remain straight but suddenly diminished in diameter before reaching the tip. Dorsocentrals 
sometimes bent crescent-like. Viable in homozygotes. Very variable but seldom overlapping wild type. A fair parallel to hook in D. melanogaster, the latter being, however, a recessive.

orange (or).-April 1948, in four different wild chromosomes from Mogi, state of São Paulo. Eyes scarlet red (Ridgway, plate I) in young flies,
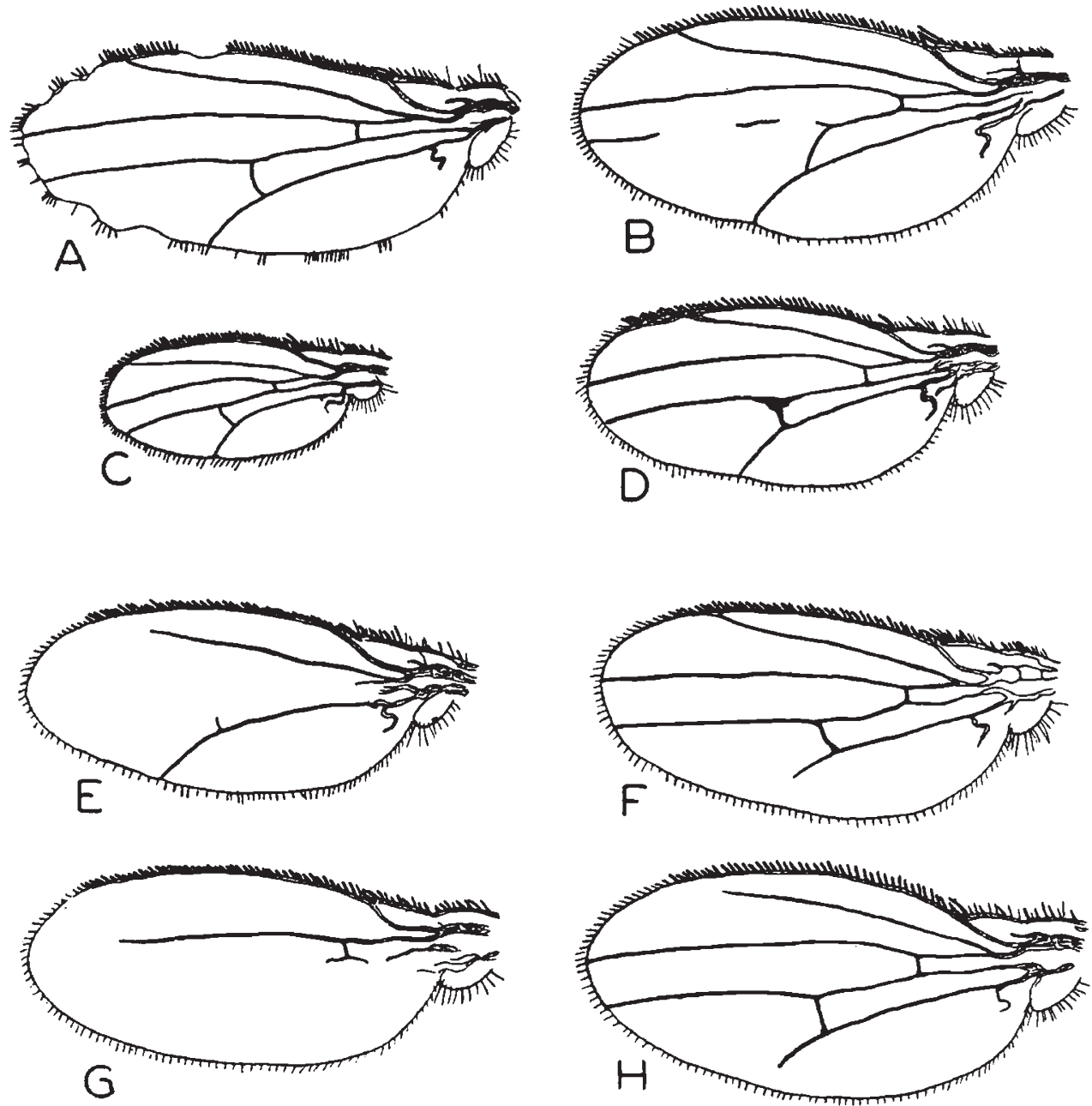

Fig. I.-Some of the wing mutants in Drosophila willistoni. A-cut; B-Incomplete; $\mathrm{C}$-miniature ; D-Delta ; $\mathrm{E}$-Incomplete veinlet; $\mathrm{F}$-short ; $\mathrm{G}$-reduced veins ; $\mathrm{H}-\mathrm{Radius}$ Incompletus. Magnification is similar in all figures.

darkening with age so that classification uncertain in old flies. A probable parallel to cinnabar in $D$. melanogaster and orange in $D$. pseudoobscura and D. persimilis.

pink-wing (pw).--August 1945, spontaneous. Eyes grenadine-red (Ridgway, plate II), age change slight. Body smaller than normal, wing somewhat shortened, wavy or crumpled, wing veins thickened and sometimes plexate. Thorax and abdomen reduced in size relatively more than the 
head. The rows of acrostichal bristles near the middle of the thorax approximated and somewhat disarranged, the outer rows more widely spaced. Viability rather low. Testis sheath transparent. Homozygous females sterile. The remarkable manifold effect of this mutant resembles, judging from description, that of pink-wing in $D$. melanogaster, but homology is uncertain.

plexus $(p x)$.-August 1946, spontaneous. Wing veins branched or plexate (fig. I). A probable homologue of plexus in $D$. melanogaster.

purple ( $p r)$.-October 1949, in a wild chromosome from Goyaz. Eye colour dragon's blood red (Ridgway, plate XIII) in young flies, darkening with age to about Madder brown (Ridgway, plate XIII). Ocelli coloured. Testis sheath yellow. A possible parallel to purple of $D$. melanogaster. Possibly the same as red of Ferry, Lancefield and Metz (1923).

scabrous (sca).-March 1948 , in a wild chromosome from São Paulo. Eyes strongly rough, frequently with bulging giant facets which result from fusion of two or more normal ones. Acrostichal hairs in 8 rows, the outer rows containing the dorsocentral bristles. Some thoracal bristles bent or reduplicated. Although seemingly not identical, a probable homologue of scabrous in $D$. melanogaster.

Shaven $(S v)$.- May 1945, in an X-rayed culture. In homozygotes most bristles and many microchaetæ small or absent. The basal rings are usually preserved, and sometimes contain a hair-like rudiment of the bristle. Some of the bristles are, however, present and normal in size. In heterozygotes the manifestation is much weaker, only a few bristles are absent but most of them shorter and thinner than normal. Resembles shaven in the fourth chromosome of $D$. melanogaster, but may also be compared to abbreviated, Bristle, chaetelle, and reduced in the second chromosome of the same species. Homology is, accordingly, ambiguous.

Star $(S)$.- March 1945, in an X-rayed culture ; a recurrence in October 1945, spontaneous. Eyes smaller than normal, surface rough, with a slight gleam as though covered with a liquid. The first occurrence was associated with a Y-II translocation, but a crossover obtained in an XXY female freed $S$ from the translocation. Lethal when homozygous. A possible parallel of Star in D. melanogaster.

straw (stw).- September 1949, in a wild chromosome from Goyaz. Bristles, hair, and wings yellow, body colour yellowish but not as distinct from normal as that of the bristles. Penetrance complete. A good parallel of straw in D. melanogaster.

\section{MUTANTS OF THE THIRD LINKAGE GROUP}

aristapedia $\left(s s^{a}\right)$.--May 1944 , in an X-rayed strain of Rio de Janeiro origin. Recurrence in January 1948 , in a strain of Belem origin, spontaneous. Aristæ transformed into leg-like organs. Variable, often asymmetrical, and overlaps wild type. The Rio allele is more penetrant than the Belem one, and behaves in heterozygotes with Delta as though the latter were a deficiency for $s s^{a}$. Rio homozygotes sterile as males. An excellent parallel to aristapedia in $D$. melanogaster.

bithorax $(b x)$.- July 1946, spontaneous. Metathorax with bristles and hairs resembling those on the mesothorax. A probable homologue of bithorax or bithoraxoid in D. melanogaster. 
brief $(b f)$.- September 1949, in a wild chromosome of Goyaz origin. Bristles and hairs thin and shorter than normal. Body slightly smaller. Females sterile, males fertile. A possible parallel to brief in $D$. melanogaster. claret $(c a)$.-September 1949, in a wild chromosome of Belem origin. Eyes grenadine red (Ridgway, plate II) in young flies, darkening with age to about dragon's blood red (Ridgway, plate XIII). Testis sheath yellow. A possible parallel to claret in $D$. melanogaster.

Delta $(\Delta)$.-April 1944, in an X-rayed culture, and several recurrences, some of the latter associated with chromosomal aberrations. This is one of the commonest mutant types obtained in X-ray treated flies. Wing veins, especially the second longitudinal one, thickened and expanded at the wing margin. Posterior crossveins thickened and expanded into deltas at the ends. Lethal when homozygous. An excellent parallel to Delta in D. melanogaster and in other species.

karmoisin (kar).-May 1949, in a wild chromosome from Cruzeiro do Sul, Acre. Eyes scarlet (Ridgway, plate I) in young flies, darkening with age to about normal colouration. A recurrence in June 1949 , in a wild chromosome from Paraná. Homology ambiguous-among the mutants in the right limb of the third chromosome of $D$. melanngaster, karmoisin and cardinal are similar.

loboid $(l d)$.- July 1945, in an X-rayed culture. Eyes rough and misshapen in various ways. Most often the eye is consiricted or divided by a cleft beginning at the posterior margin and progressing towards the anterior one. Often the eyes are bulging or drawn out into outgrowths covered with bristles and resembling an antenna. Occasionally overlaps normal. A good parallel to loboid in $D$. melanogaster.

pink $(p)$.- March 1948 , in a wild chromosome of São Paulo origin. Eyes dragon's blood red (Ridgway, plate XIII) in young flies, approaching normal colouration in old ones. Testis sheath lighter than normal. Homology ambiguous.

Radius Incompletus $(R i)$.- May I944, in an X-rayed culture. Second and fifth longitudinal veins fail to reach the margin (fig. I). In compound with Delta, the effects of the two mutants almost cancel each other, the flies being usually identifiable as very weak Deltas. Homozygote seldom survives as a fly almost wholly devoid of bristles and hair and only the costa and the third longitudinal vein complete in the wing, other veins reduced to mere stubs. No clear analogues in $D$. melanogaster.

Roof $(R f)$.- July 1945, in an X-rayed culture. The wings are held roof-like. The scutellum distinctly longer and relatively narrower than normal. A doubtful parallel to roof in D. melanogaster. Males sterile.

tardigrade $(t d)$.- May 1945, in an X-rayed culture. Tarsi rudimentary or absent, tibiæ and femora somewhat shortened. Variable. The degree of reduction of the tarsi usually greatest on the front legs, on middle and hind legs tarsi usually present but malformed in various degrees. Resembles tardigrade and combined of Ferry, Lancefield and Metz (1923) but not identical with either. Resembles the sex-linked mutant chilblained- $b$ in D. melanogaster.

\section{LINKAGE MAPS}

The chromosomal complement of all known species of the willistoni group, and of most species of the saltans group, consists of a pair of metacentric X- or Y-chromosomes (indistinguishable at metaphase), 
TABLE I

Summary of linkage data. Three point crosses

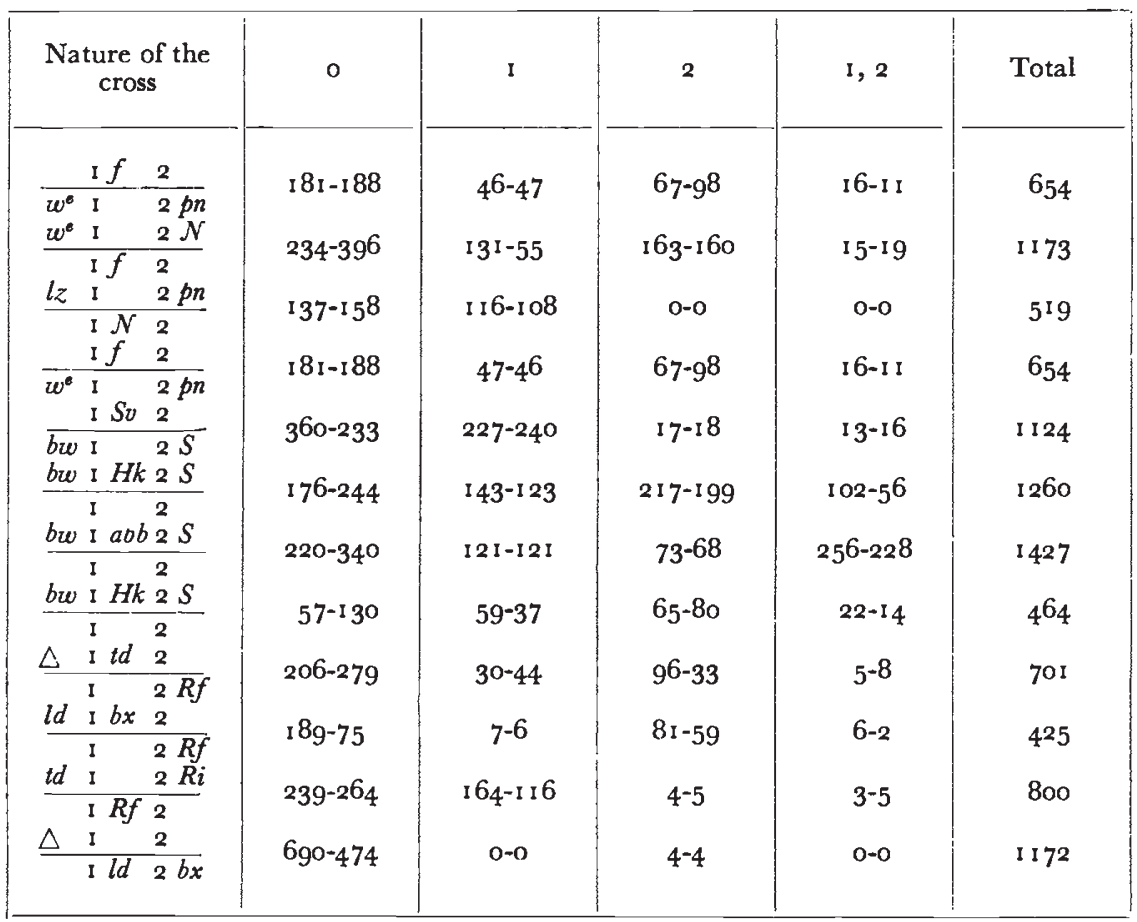

TABLE 2

Summary of linkage data. Four point crosses

\begin{tabular}{|c|c|c|c|c|c|c|c|c|c|c|c|c|}
\hline & $\begin{array}{r}\text { Nature } \\
\text { cr }\end{array}$ & $\begin{array}{l}\text { e of } t \text { t } \\
\text { oss }\end{array}$ & & 0 & I & 2 & 3 & 1,2 & I, 3 & 2,3 & $I, 2,3$ & Total \\
\hline & $\mathbf{I}$ & $2 \mathrm{se}$ & & \multirow{3}{*}{$\begin{array}{l}140-102 \\
54^{-1} 4^{6}\end{array}$} & \multirow[b]{2}{*}{$49-49$} & \multirow[b]{2}{*}{$9^{-I I}$} & \multirow{3}{*}{$\begin{array}{l}53-45 \\
27-84\end{array}$} & \multirow{2}{*}{$6-3$} & \multirow[b]{2}{*}{$27-23$} & \multirow[b]{2}{*}{$2-I$} & \multirow[b]{2}{*}{$0-0$} & \multirow{2}{*}{$5^{20}$} \\
\hline$s c$ & $\begin{array}{l}\text { I } I n c \\
\text { I }\end{array}$ & $\begin{array}{l}2 \\
2 \mathrm{co}\end{array}$ & $\begin{array}{l}3 r u \\
3\end{array}$ & & & & & & & & & \\
\hline $\begin{array}{l}s c \\
l z\end{array}$ & I $I n c$ & $2 p n$ & $\begin{array}{l}3^{r u} \\
3\end{array}$ & & $\begin{array}{l}99-10 \\
40-65\end{array}$ & $0-0$ & & $0-0$ & $30-29$ & $0-0$ & $0=0$ & 479 \\
\hline & $\begin{array}{l}\text { I } s C \\
\text { I } e C\end{array}$ & $\begin{array}{l}2 \\
2\end{array}$ & $\begin{array}{l}3 \operatorname{Inc} \\
3\end{array}$ & $303-355$ & $40-0$ & $153^{-1} 4^{0}$ & $0-0$ & $3-7$ & $1-0$ & $0-2$ & $0-0$ & I ob9 \\
\hline $\begin{array}{l}w^{e} \\
w^{e}\end{array}$ & I & $\begin{array}{l}2 p n \\
2 s n\end{array}$ & $\begin{array}{l}3 s h \\
3 p n\end{array}$ & $220-131$ & $4-04$ & $33^{-122}$ & $10-15$ & $9=0$ & $5-4$ & $10-20$ & $3^{-1}$ & 700 \\
\hline & $\begin{array}{l}\text { I } y \\
1\end{array}$ & $\begin{array}{l}2 \\
2\end{array}$ & $\begin{array}{l}3 \\
3 v e\end{array}$ & $10-1 / 0$ & $2+15$ & $35^{-11}$ & $y<-43$ & $0-1$ & $2-9$ & $1-2$ & $0-0$ & 488 \\
\hline $\begin{array}{l}\text { Inc } \\
w^{\circ}\end{array}$ & $\begin{array}{l}\text { I } S e \\
\text { I }\end{array}$ & $\begin{array}{l}2 r u \\
2 s c\end{array}$ & $\begin{array}{l}3 \\
3 r u\end{array}$ & $217-83$ & $8-36$ & $47-68$ & $8-4$ & $0-2$ & $3^{-1}$ & $0-0$ & $0-0$ & 477 \\
\hline & $\begin{array}{l}\text { I } y \\
\text { I } s n\end{array}$ & $\begin{array}{l}2 \\
2\end{array}$ & $\begin{array}{l}3 \\
3 \\
\end{array}$ & $123-02$ & $10-21$ & $1-0$ & $01-3^{2}$ & $0-1$ & $2-5$ & $0=1$ & $0=0$ & 359 \\
\hline$w^{e}$ & $\begin{array}{l}\text { I } a b b \\
\text { I }\end{array}$ & $\begin{array}{l}2 p n \\
2 S v\end{array}$ & $\begin{array}{l}3 r u \\
3\end{array}$ & $248-102$ & $60-39$ & 33-95 & 84-60 & $12-5$ & $31-3^{2}$ & $19^{-24}$ & $5-4$ & $85^{6}$ \\
\hline bw & I $p w$ & 2 & $\begin{array}{l}3 S \\
3 S\end{array}$ & $-40-1 y^{2}$ & $74-8$ & $103^{-1} 55$ & $14^{-11}$ & $4^{2-39}$ & $10-9$ & $5^{-1}$ & $5-5$ & 1110 \\
\hline$H k$ & & $2 S v$ & & $00-177$ & $74-01$ & $5^{-1}$ & $0-10$ & $0-2$ & $0-9$ & $0=0$ & $0-0$ & 443 \\
\hline$\overline{l d}$ & $\frac{I}{\text { I } b x}$ & 2 & $\frac{3 R i}{3}$ & $327-136$ & $8-10$ & $143-225$ & $3^{0-3^{8}}$ & $I-2$ & $I-O$ & 20-I9 & $0-0$ & 960 \\
\hline
\end{tabular}


a pair of metacentric second, and a pair of acrocentric third chromosomes. The small, dot-like autosome characteristic of many Drosophila species is absent, and the salivary gland cells show only five long and no short strands (the contrary findings of Wharton, 1943, are probably inaccurate). The three linkage groups found among the mutants thus correspond to the three pairs of chromosomes. Cytogenetic study of some chromosomal aberrations (unpub.) showed that the second linkage group is carried in the metacentric autosome and the third in the acrocentric autosome.

TABLE 3

Summary of linkage data. Five point crosses

\begin{tabular}{|c|c|c|c|c|c|}
\hline \multirow{2}{*}{ Interval } & $1 w^{e} 2 s c 3^{r s 4}$ & I $w^{e} 2 l z 3 p n 4$ & $\begin{array}{lll}1 & 2 & 3 \\
I n c 4\end{array}$ & ${ }_{1} s_{2} \operatorname{Inc} 3 \quad 4$ & I $w^{e} 2 p n 3 \quad 4^{r u}$ \\
\hline & $m_{\mathrm{I}} 234^{s h}$ & $\begin{array}{llll}m_{\mathrm{I}} & 2 & 3 & 4^{\text {sh }}\end{array}$ & $w^{2} 1 \operatorname{sc} 2 c t 3 \quad 4 p n$ & $\begin{array}{lll}w^{e} 1 & 2 & 3 p n 4 r u\end{array}$ & $\begin{array}{lll}m 1 & 2 & 3 s h 4\end{array}$ \\
\hline 0 & $225-169$ & $197^{-1}$ I 2 & $235-215$ & $246-230$ & $3^{11} 1-37^{2}$ \\
\hline I & $4-3$ & $3-0$ & $47-40$ & $42-31$ & $5^{-0}$ \\
\hline 2 & $24-14$ & $4-4$ & $126-137$ & II I -141 & $264-179$ \\
\hline 3 & $207-123$ & $121-77$ & $3-1$ & $3-0$ & $120-115$ \\
\hline 4 & $0-6$ & $87-49$ & $0-1$ & $83-96$ & $15-5$ \\
\hline 1,2 & $0-0$ & $0-1$ & $13-9$ & $4-8$ & $0-1$ \\
\hline 1,3 & $0-1$ & $0-4$ & $0-2$ & $0=0$ & $1=0$ \\
\hline 1,4 & $0-0$ & $1-4$ & $0-2$ & I I -6 & $2-2$ \\
\hline 2,3 & $10-21$ & $1-0$ & $2-0$ & $2-0$ & $99-43$ \\
\hline 2,4 & $4=0$ & $0-0$ & $1=0$ & $40-44$ & $12-5$ \\
\hline 3,4 & $0-0$ & $63-24$ & $0-0$ & $0-0$ & $0=0$ \\
\hline $1,2,3$ & $0-0$ & $0-0$ & $0-0$ & $0-0$ & $I=0$ \\
\hline $1,2,4$ & $0-0$ & $0-0$ & $0-0$ & 0.2 & $2-0$ \\
\hline $1,3,4$ & $0-0$ & $0=0$ & $0-0$ & $0=0$ & $0-0$ \\
\hline $2,3,4$ & $0-1$ & $0-0$ & $0-0$ & $0-0$ & $0=0$ \\
\hline Total & 812 & $75^{2}$ & 834 & 1100 & 1554 \\
\hline
\end{tabular}

The linkage data are summarised in tables $\mathrm{I}-3$, and linkage maps of the chromosomes constructed on the basis of these data are shown in fig. 2. In contrast to the high variability in crossover percentages found in different experiments on $D$. prosaltans (see the companion paper of Spassky, Zimmering and Dobzhansky), the linkage values in $D$. willistoni are reasonably constant. This makes the construction of the linkage maps easier than in the former species.

\section{DISCUSSION}

Our data confirm the conclusion of Lancefield and Metz (1922) and Sturtevant and Novitski (I94I) that the two arms of the metacentric X-chromosome of Drosophila willistoni more or less correspond to the X-chromosome and to the left limb of the third chromosomes respectively in $D$. melanogaster. Indeed, the first fifty units of the linkage map of the $\mathrm{X}$ of $D$. willistoni (fig. 2) contain the mutants miniature, white, lozenge, yellow, scute, forked and singed, which parallel the similarly named sex-linked mutants in D. melanogaster (Bridges and Brehme, 1944) to such an extent that the assumption that they are 
homologous is probable. The similarity between the mutants echinus, cut, prune and Notch in the two species is not so striking as in the cases named above. Echinus of $D$. willistoni is, in fact, more like echinoid in the second chromosome than the sex-linked echinus in

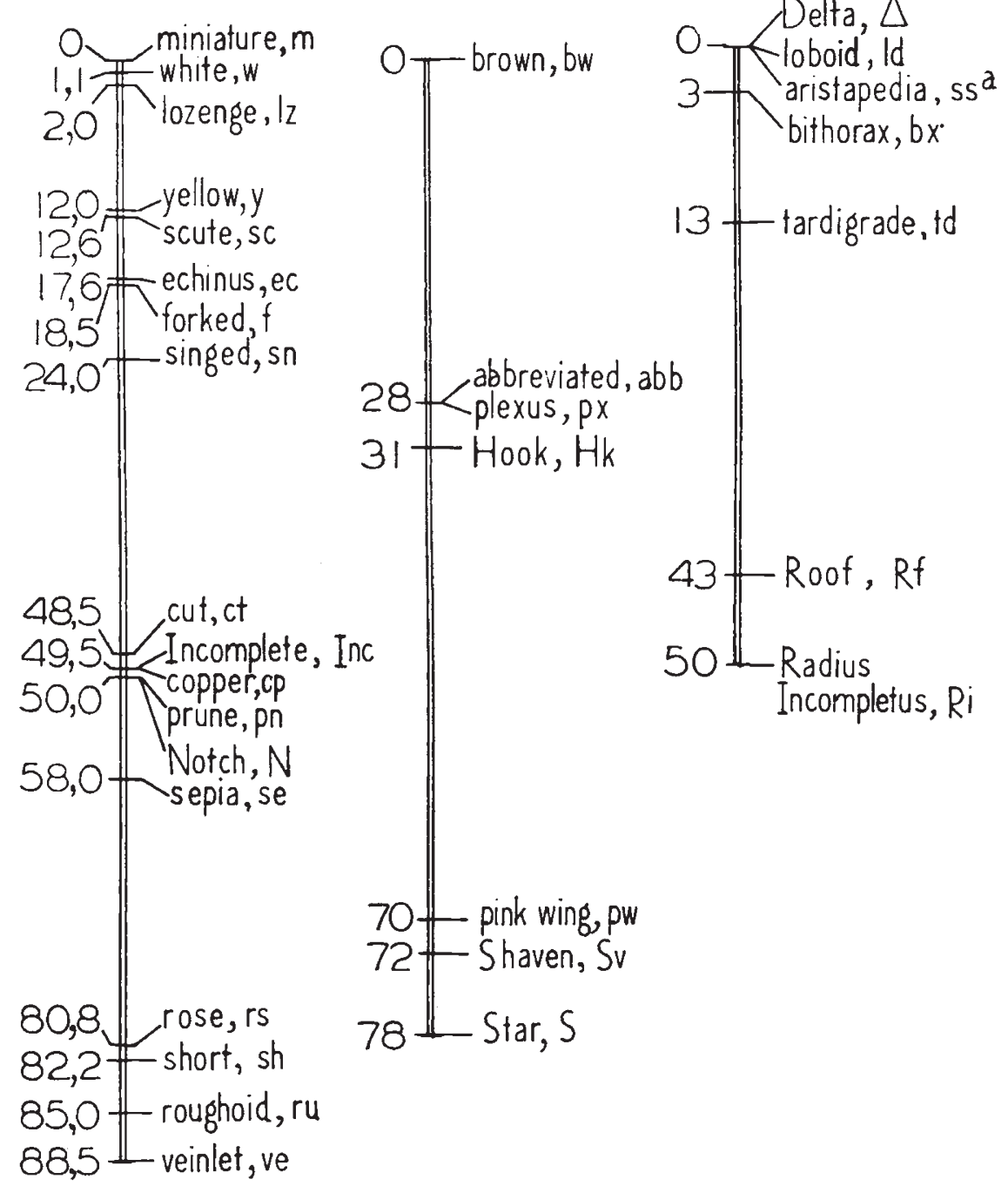

Fig. 2.-Linkage maps of the X-chromosome (left), second chromosome (middle), and third chromosome (right) of Drosophila willistoni.

D. melanogaster. Nevertheless, the assumption of homology is still the simplest one. The remainder of the linkage map (loci from sepia to veinlet) of the $\mathrm{X}$-chromosome of $D$. willistoni contains sepia, roughoid and veinlet, all of which more or less resemble mutants in the left limb of chromosome III of $D$. melanogaster, and rose and short, the homology of which is obscure. Only the mutant stubbloid suggests a 
homology with the right limb of the third chromosome of $D$. melanogaster ; its location on the map of the $\mathrm{X}$ of $D$. willistoni is unknown since it has been obtained in a strain from Southern Brazil which gives no crossing over in the X-chromosome in hybrids with the Belem strain. It may be that a small translocation has taken place in the phylogeny of the species, or the mutants may be " mimics" rather than homologous.

The second chromosome of $D$. willistoni contains mostly the same genes as the second chromosomes of $D$. melanogaster and $D$. prosaltans, and the third plus the fourth chromosomes of $D$. pseudoobscura. This is suggested especially by the mutants brown, Emarginate, Hook, orange, plexus, scabrous and straw. The mutants pink-wing, purple and Star are less conclusive but still compatible with the above interpretation. Abbreviated and Shaven suggest, however, genes in the right limb of the third and in the fourth chromosomes respectively of $D$. melanogaster. Whether small translocations or "mimics" are involved, is an open question.

The third chromosome of $D$. willistoni should, then, be homologous mainly to the right limb of the third chromosome in D. melanogaster, and to the second chromosome of $D$. pseudoobscura. The best evidence of this comes from the mutants aristapedia, bithorax, Delta and loboid. The mutants claret, karmoisin, and pink are compatible with this interpretation. Radius Incompletus, Roof and tardigrade are ambiguous.

Comparison of $D$. willistoni with $D$. prosaltans shows that the distribution of genes among the chromosomes is similar in the two species: the $\mathrm{X}$, the second, and the third linkage groups of the former correspond to the $\mathrm{X}$, the second, and the third chromosomes of the latter. Now, these species are representatives of two species groups (willistoni and saltans groups) of the subgenus Sophophora of the genus Drosophila. Both groups are characteristically Neotropical in their distribution (Patterson and Wheeler, 1949). Apart from willistoni and prosaltans, some twelve species of these groups have been examined cytologically, and all but one of them have the same metaphase chromosome group : two large metacentric and a acrocentric chromosome pair, one of the metacentrics being the X. It seems, then, that the three linkage groups have tended to preserve their identities in the evolution of the Neotropical Sophophora.

Two other species groups belonging to the subgenus Sophophora are the melanogaster group, which is characteristically Oriental, and the obscura group (including affinis), which is Holarctic in distribution (Patterson and Wheeler, 1949). A variety of metaphase chromosome patterns are known in these groups, none of them identical with that in the Neotropical forms. However, the same metacentric autosome seems to occur in the willistoni, saltans and melanogaster groups (corresponds to the second linkage group in D. willistoni, D. prosaltans, $D$. melanogaster and D. simulans, and to the third in D. ananasse and 
D. montium (see Sturtevant and Novitski, I94 I, for further references). The large metacentric X-chromosome of the Neotropical willistoni and saltans groups does not occur in the melanogaster group, but it is found in a majority of species of the Holarctic obscura group (D. pseudoobscura, $D$. persimilis, $D$. miranda, probably all species related to $D$. affinis, but not in D. subobscura and some Old World species related to the last-named one).

Finally, a small dot-like autosome is present in many species of several subgenera of Drosophila. In the subgenus Sophophora, this autosome occurs in all species of the obscura group and in some of the melanogaster group. In the Neotropical willistoni and saltans groups it is never present, and our data suggest that it has become broken up and incorporated into other linkage groups. In $D$. prosaltans a part of it is included in the X-chromosome, but at least one locus appears to lie in the third chromosome. The situation in $D$. willistoni is unclear; the second-chromosome mutant Shaven in this species is not a reliable homologue of the similarly named mutant in the dot-like chromosome in $D$. melanogaster.

Closely related species of Drosophila, and even varieties of the same species, may differ considerably in the distribution of genes among the linkage groups (Stone and Patterson, I947; Ward, 1949). Nevertheless, the correlations pointed out above between chromosome structure, systematic position, and geographic distribution indicate that, in the subgenus Sophophora and particularly in its Neotropical representatives, chromosome fusion and fragmentation have been, on the whole, infrequent in the phylogeny. Despite the probable occurrence of small translocations, the linkage groups preserve their essential integrity for long periods of time.

The gene arrangement inside the chromosomes is, on the contrary, frequently altered by inversions. Comparison of the linkage maps of the X-chromosome of $D$. willistoni obtained by ourselves (fig. 2), and by Lancefield and Metz (1922) discloses appreciable differences. Their maps show the genes forked and singed (stubby) some 30 units apart at the end of the chromosome, while yellow and scute are in the middle portion of their linkage map ; we found forked and singed only 6 units apart, and yellow and scute between forked and the end of the map. These disagreements are almost certainly due to our mutants having been obtained in a strain of Brazilian origin, whereas Lancefield and Metz worked with a strain from Cuba. These strains probably differed by inversions.

The variability of the gene arrangement within a species may seem to make comparisons of different species meaningless. Actually some associations between neighbouring genes are quite persistent. Thus, yellow and scute are adjacent in species as diverse as $D$. willistoni, $D$. prosaltans, $D$. pseudoobscura, $D$. persimilis, D. melanogaster, D. simulans, $D$. ananasse and $D$. virilis, although not in $D$. affinis and $D$. hydei (cf. Sturtevant and Novitski, I94I). On the other hand, white is 
close to yellow and scute in $D$. melanogaster and $D$. simulans, somewhat farther apart in $D$. willistoni and $D$. pseudoobscura, and very far apart in $D$. prosaltans, $D$. ananasse and $D$. virilis.

The length of the linkage map of a chromosome depends, in part, on the number of loci known in a linkage group. The more mutant loci are placed, the greater the probability that loci near the ends of the chromosome will be included, and the greater the chance that the total length of the linkage map will be known. Despite this source of error, comparison of the published linkage maps for different species of Drosophila suggests the existence of genetic differences in the amount of recombination in homologous chromosomes in these species. In species with large metacentric X-chromosomes, the known map lengths of the $\mathrm{X}$ are some 90 units in $D$. willistoni, 100 in $D$. prosaltans, $\mathrm{I}_{5}$ in $D$. persimilis and $\mathrm{I} 80$ in D. pseudoobscura (Beers, 1937 ; Sturtevant and Tan, I937 ; Spassky. Zimmering and Dobzhansky, 1950, and the present article). In species with acrocentric or small metacentric $\mathrm{X}$-chromosomes, the map length of the $\mathrm{X}$ is 66 units in $D$. melanogaster and $D$. simulans, I 6 in $D$. ananassa, 150 in $D$. subobscura and 170 in $D$. virilis (Bridges and Brehme, I944; Chino, I936; Moriwaki, I938; Spurway, 1945). As stated above, only parts of the large metacentric X's correspond to the short metacentric or to acrocentric X-chromosomes. Approximate estimates of the length of these parts are 55 units in $D$. willistoni, between 60 and 70 in $D$. prosaltans, about 6 o in $D$. persimilis and 73 in $D$. pseudoobscura.

The large metacentric autosome is at least 59 units long in $D$. prosaltans, 78 in D. willistoni, 94 in D. ananasse and 108 in D. melanogaster. The combined lengths of the two acrocentric autosomes which correspond to the above metacentric ones are 137 units in $D$. pseudoobscura and 379 in $D$. virilis. Finally, the acrocentric autosome is $5^{\circ}$ units long in $D$. willistoni, $5^{2}$ in $D$. prosaltans and 2 10 in $D$. virilis. Comparison of these figures suggests the conjecture that small amounts of recombination are characteristic of tropical species, and high amounts of those distributed chiefly in the temperate zones $(D$. willistoni, $D$. prosaltans and $D$. ananass $a$ are tropical $; D$. melanogaster and $D$. simulans now cosmopolitan but originally probably subtropical, while $D$. pseudoobscura, $D$. persimilis and $D$. virilis are temperate). More data are obviously necessary to test the validity of this conjecture.

\section{SUMMARY}

Drosophila willistoni has a large metacentric X-chromosome, a large metacentric second chromosome and a acrocentric third chromosome. Three linkage groups corresponding to these chromosomes are established.

Comparison of the mutants indicates that the chromosomes of D. willistoni contain largely the same genes as the cytologically similar chromosomes of $D$. prosaltans, although the sequences of these genes 
within the chromosomes are quite different, because of the repeated occurrence of paracentric inversions in the phylogeny. Homologies with the chromosomes of other species of Drosophila are suggested. Certain correlations between the systematic position, geographic distribution, and the chromosome contents in species of the subgenus Sophophora of Drosophila appear possible. The two Neotropical sections of the subgenus, namely, the willistoni and the saltans species groups, have very similar chromosomes. The large metacentric X-chromosome characteristic of these sections appears also in most species of the Holarctic obscura species group, but not in the Oriental melanogaster species group. Conversely, the large metacentric autosome of the willistoni and saltans groups is shared with melanogaster but not with the obscura group. The acrocentric autosome of the willistoni and saltans groups occurs also in the obscura group, but in the melanogaster group it is joined with a chromosome which in the willistoni, saltans, and in most species of obscura group is a part of the X-chromosome.

The amounts of recombination which occur in corresponding chromosomes of different species vary greatly. A suggestion is made that tropical species have less recombination than species which inhabit temperate lands.

\section{REFERENCES}

BEERS, C. V. $\quad$ I 937 .

Linkage groups in Drosophila pseudoobscura race B.

Geneitics, 22, 577-586.

BRIDGES, C. B., AND BREHME, K. S. I944.

The mutants of Drosophila melanogaster.

Carnegie Inst. Washington, Publ. 552, I-257.

CHINo, M. 1936.

The genetics of Drosophila virilis.

Fap. F. Genet., 12, 187-210, 257-277.

FERRY, R. M., LANCEFIELD, R. C., AND METZ, C. W. I923.

Additional mutant characters in Drosophila willistoni.

7. Heredity, $14,373-384$.

LANCEFIELD, R. C., AND METZ, C. W. I 922.

The sex-linked group of mutant characters in Drosophila willistoni.

Amer. Nat., 56, 2 I I-24I.

MORIWAKI, D. I $93^{8}$.

The genetics of some mutant characters in Drosophila ananasse.

Jap. J. Genet., I4, I-22.

PAtterson, J. T., AND Wheeler, M. R. 1949 .

Catalogue of described species belonging to the genus Drosophila, with observations on their geographical distribution.

Univ. Texas Publ., 4920, 207-233.

RIDGWAY, R. I 912.

Color standards and color nomenclature. Washington. 
SPURWAY, H. 1945.

The genetics and cytology of Drosophila subobscura. I. Element A.

7. Genet., 46, 268-286.

SPASSKY, B., ZIMMERING, S., AND DOBZHANSKY, TH. I950.

Comparative genetics of Drosophila prosaltans.

Heredity, 4, 189-200.

STONE, w. s., AND PATTERSon, J. T. 1947.

The species relationships in the virilis group.

Univ. Texas Publ., 4720, 157-16o.

STURTEVANT, A. H., AND NOVITSKI, E. 1941 .

The homologies of the chromosome elements in the genus Drosophila.

Genetics, 26, 517-541.

STURTEVANT, A. H., AND TAN, c. c. 1937.

The comparative genetics of Drosophila pseudoobscura and D. melanogaster.

7. Genet., 34, 415-432.

WARD, C. L. 1949 .

Karyotype variation in Drosophila.

Univ. Texas Publ., 4920, 70-79.

WHARTON, L. T. 1943 .

Analysis of the metaphase and salivary chromosome morphology within the genus Drosophila.

Univ. Texas Publ., 4313, 282-327. 